\title{
PREFACE
}

\section{Science's gem: diamond science 2009}

\section{Guest Editors}

\author{
Alison Mainwood \\ Department of Physics, \\ King's College London, \\ Strand London WC2R $2 L S$, \\ $U K$
}

\section{Mark E Newton \\ Department of Physics, \\ University of Warwick, \\ CV4 7AL, UK}

\section{Marshall Stoneham \\ London Centre for \\ Nanotechnology and \\ Department of Physics and Astronomy, \\ University College London, \\ 17-19 Gordon Street. \\ London WC1H OAH, UK}

Natural diamond has been valued for its appearance and mechanical properties for at least two thousand years. As a gem stone diamond is unsurpassed. However, scientific work, especially in the last 20 years, has demonstrated that diamond has numerous surprising properties and many unique ones. Some of the extreme properties have been known for many years, but the true scale of diamond's other highly desirable features is still only coming to light as control in the synthesis of diamond, and hence material perfection, improves. The ultimate prize for man-made diamond is surely not in the synthesis of gem stones, but in delivering technological solutions enabled by diamond to the challenges facing our society today.

If the special properties are to be exploited to their full potential, at least four crucial factors must be considered. First, there must be sufficient scientific understanding of diamond to make applications effective, efficient and economical. Secondly, the means of fabrication and control of properties have to be achieved so that diamond's role can be optimised. Thirdly, it is not enough that its properties are superior to existing materials: they must be so much better that it is worth initiating new technologies to exploit them. Finally, any substantial applications will have to address the society's major needs worldwide. The clear technology drivers for the 21 st century come from the biomedical technologies, the demand for energy subject to global constraints, and the information technologies, where perhaps diamond will provide the major enabling technology [1].

The papers in this volume concern the solid state physics of diamond, and primarily concern the first two factors: understanding, and control of properties. They address many of the outstanding basic problems, such as the identification of existing defects, which affect the material's properties, both desirable and less so. Regarding future substantial applications, one paper discusses diamond's exceptional properties for quantum information processing [2], a topic on which there have been many recent papers, and where a diamond colour centre single photon source is already commercially available. Biomedical applications of diamond are recognised, partly tribological and partly electrochemical, but lie outside the present group of papers. Processing and controlling diamond surfaces and interfaces with other materials in their environment are critical steps en route to exploitation. Boron-doped diamond has already found application in electro-analysis and in the bulk oxidation of dissolved species in solution [3]. Energy-related applications - ranging from high-power electronics [3] to a potential first wall of fusion reactors [4] - are further exciting potential applications. Even small and ugly diamonds have value. Their mechanical properties [5] dominate, with significant niche applications such as thermal sinks. The major applications for diamond to date exploit only a fraction of diamond's special properties: visual for status diamonds, and mechanical for working diamonds.

Diamond physics reaches well beyond the usual laboratory, to the geological diamond formation processes in the Earth's mantle. Characterization of natural gem diamonds $[6,7]$ is one part of the detective story that allows us to understand the conditions under which they formed. It was only half a century ago that the scientific and technological challenges of diamond synthesis were met systematically. Today, most of the recent research on diamond has concentrated 
on synthetics, whether created using high pressure, high temperature (HPHT) techniques or chemical vapour deposition (CVD). The HPHT synthesis of diamond has advanced dramatically $[8,9]$ to the extent that dislocation birefringence [10] can be largely eliminated. In silicon technology, the elimination of dislocations was a major step in microelectronics. Now, even diamond can be synthesised containing virtually no dislocations. The understanding of the critical processes that are involved in CVD diamond growth are becoming clearer. Two papers in this issue model it on a microscopic scale $[11,12]$, and a further two explore the practical techniques $[13,14]$ in order to lead to improvement in deposition techniques. Diamond is emerging as an engineering material [3], with its cost no longer regarded as prohibitive even for some large-scale uses, such as the fusion reactor first wall.

It is striking how few useful dopants can be put into diamond in a controlled way. The studies reported here, whether theory or experiment, concentrate on phosphorus [14] as the donor, and demonstrate that boron (although deep in semiconductor terms,) can act as the acceptor [3] in practical devices. Other impurities, with deeper levels, such as nitrogen [15], with the muon as an honorary hydrogen [16], are studied in depth. Here, many of the characterization techniques developed over several decades have been brought to bear, to attempt to quantify impurities and defects and ultimately assist in improving the crystal quality $[17,18,15]$. However, further, more novel techniques such as reflection anisotropy spectroscopy [19] and luminescence lifetime mapping [20] have been introduced to diamond in this issue, and one can see how such techniques might play a crucial role in areas such as systems for quantum information processing.

The presence and migration of radiation damage defects [21,22], vacancies and interstitials, and vacancy clusters can dramatically influence the exploitable properties of diamond [23,24]. It is now apparent that charge traps not only impact on electrical properties, but also on the colour of diamond and that thermo-chromic and photo-chromic effects are more common than previously thought $[25,23]$. Combinations, like the negatively charged nitrogen-vacancy centre, have proved impressive in quantum information studies [26]. But diamond has yet to benefit from the sort of dopant engineering that has helped silicon to become ubiquitous. It is becoming clear that because of the deep ionisation energies of the dopants that can be incorporated into diamond, conventional semiconductor physics can only be applied at high temperatures; rather different technologies have to be exploited to ensure that diamond's potential for devices is fulfilled. There are technical improvements which need to be made: the elimination of defects that trap carriers, cause de-coherence, affect the colour or strength, or have other serious effects in the relevant application, and the development of robust ohmic contacts [27].

The material developments of the last 50 years include silicon becoming the semiconductor of choice, many new and better-developed polymers, the transformation of communications by silica-based optical fibres, and the emergence of synthetic diamond. Could diamond's special virtues yield major new opportunities? Its optical properties are exceptional, usually in desirable ways (high refractive indices can create indirect problems). The mechanical properties are truly outstanding, again usually in desirable ways (adhesion can be challenging). The thermal properties are similarly exceptional, with a thermal conductivity that exceeds copper. Diamond withstands aggressive environments, including extremes of $\mathrm{pH}$. Its carrier mobility can be phenomenal, and electron emission can be excellent. Moreover, diamond can be compatible with silicon electronics, even if the involvement of a second material is inconvenient. Here the problems start. Even limited developments could be significant. For instance, the ability to control the populations of the various N, B, P and vacancy centres would open up potentially unique optoelectronic and spintronic opportunities. Control of diamond's properties is difficult, but this is where basic research can help 
(using all the techniques explored in this issue, and more). It is barely practical to create n-type diamond, but unipolar devices, exploiting excellent quality boron doped p-type material, can be designed [3]. Electrical contacts can be tricky to fabricate, but progress is being made here [3,27]. Diamond is perceived as unacceptably expensive, but for a high-quality device for an exceptional environment, this is not a problem.

Carbon-based electronic materials are strikingly diverse. They include diamond, graphite, nanotubes and buckyball structures, amorphous carbons, and nanodiamond. Add hydrogen and one has a range of diamond-like carbons and the wealth of organics. Such carbon-based materials include small molecules and polymers: impressive insulators, semiconducting and conducting polymers, switchable forms, superconducting and magnetic forms, and some with the highest electrical conductivities of any material. Diamond-like carbons can have controllable mechanical properties from the viscoelastic to the highly rigid. Photochemistry brings opportunities for novel processing methods. Even water-based processing may sometimes be possible (alas, not for diamond), and additional tools like self-organisation of organic molecules on surfaces have been demonstrated. The best carbons have impressive, sometimes supreme, performances, including the mobility and optical properties of diamond, spin-conserving transport in carbon nanotubes, and electron emission. For almost all measures of performance, there is some carbon-based material that performs better than silicon. Might hybrid carbon-based materials be more successful even than silicon [28]? Should we think less about 'diamond' and more about the integration of diamond as one component of carbon electronics? Device fabrication needs lithography optics and resists, and processing at the anticipated smaller scales may well exploit new electronic excitation methods. Alternative dielectrics and interconnect materials introduce new compatibility issues, and there are further varied constraints from displays, spintronic components, electron emitters or transparent conductors. Could the many carbon-based materials with interesting functional properties lead to a new class of alternative systems?

This collection of papers was brought together to celebrate 60 years of conferences sponsored by the De Beers Group of companies on the science and technology of diamond. The transformation of diamond science and technology over those 60 years can be seen in varied ways. First, there has been a series of books stimulated by the conferences [29-31] complementing numerous other more recent texts on diamond (e.g. [32]). These show a striking evolution from the early pioneering studies of tribology, radiation damage, and thermal and optical properties to a wider range of electronic properties, spectroscopies, and characterization from the macroscopic to nanoscopic scales, as well as the now almost universal dialogue between experiment and theory. Secondly, new experimental and theoretical techniques have emerged, many of which are featured in the papers in this issue. Thirdly, there is a range of new technologies only made possible because of the catalytic role of the conferences. These include the spectroscopies that distinguish natural from synthetic or treated diamonds in a way that earns customer confidence. There are also new customer products, like speaker domes, where success has depended on the understanding of mechanical properties at a level not commonly available. Potentially big applications, like the fusion reactor's first wall, will follow on from early radiation damage studies. Fourthly, the young scientists who have been supported over the years have now made their way in many fields, not just diamond research, but certainly including technologies that use diamond. The sponsorship of science in this field has benefited both those supported and those who provide that support. Finally, we see serious thoughts about what might be the big new technologies of the 21 st century, since these will need a fundamental understanding of materials properties and their control. There has been exceptional progress in this area, in specimen sizes, quality, and performance. These massive improvements in materials 
availability create opportunities for the major technological applications in the energy, environment, health and information technologies that will surely drive the big industrial expansions over the next decades.

\section{References}

[1] Stoneham A M 2007 Thinking about diamond (ed P Bergonzo, R Gat, R B Jackman and C E Nebel) MRS Proc. 956 1-10

[2] Stoneham A M 2008 Future Perspectives for Diamond for Physics and Applications of CVD Diamond ed S Koizumi, M Nesladek and C E Nebel (New York: Wiley-VCH)

[3] Balmer R S et al 2009 J. Phys.: Condens. Matter 21364221

[4] Stoneham A M, Matthews J R and Ford I J 2004 J. Phys.: Condens. Matter 16 S2597

[5] Liang Q, Yan C, Meng Y, Lai J, Krasnicki S, Mao H and Hemley R J 2009 J. Phys.: Condens. Matter 21364215

[6] Stachel T and Harris J W 2009 J. Phys.: Condens. Matter 21364206

[7] McNeill J, Pearson D G, Klein-BenDavid O, Nowell G M, Ottley C J and Chinn I 2009 J. Phys.: Condens. Matter 21364207

[8] Martineau P M, Gaukroger M P, Guy K B, Lawson S C, Twitchen D J, Friel I, Hansen J O, Summerton G C, Addison T P G and Burns R 2009 J. Phys.: Condens. Matter 21364205

[9] Burns R C et al 2009 J. Phys.: Condens. Matter 21364224

[10] Pinto H and Jones R 2009 J. Phys.: Condens. Matter 21364220

[11] May P W, Allan N L, Ashfold M N R, Richley J C and Mankelevich Yu A 2009 J. Phys.: Condens. Matter 21364203

[12] Butler J E, Mankelevich Yu A, Cheesman A, Ma J and Ashfold N R 2009 J. Phys.: Condens. Matter 21364201

[13] Silva F, Hassouni K, Bonnin X and Gicquel A 2009 J. Phys.: Condens. Matter 21364202

[14] Haenen K, Lazea A, Barjon J, D’Haen J, Habka N, Teraji T, Koizumi S and Mortet V 2009 J. Phys.: Condens. Matter 21364204

[15] Felton S, Cann B L, Edmonds A M, Liggins S, Cruddace R J, Newton M E, Fisher D and Baker J M 2009 J. Phys.: Condens. Matter 21364212

[16] Etmimi K M, Goss J P, Briddon P R and Gseia E M 2009 J. Phys.: Condens. Matter 21364211

[17] Moore M 2009 J. Phys.: Condens. Matter 21364217

[18] Maki J M, Tuomisto F, Kelly C J, Fisher D and Martineau P M 2009 J. Phys.: Condens. Matter 21364216

[19] Schwitters M, Martin D S, Unsworth P, Farrell T, Butler J E and Weightman P 2009 J. Phys.: Condens. Matter 21364218

[20] Liaugaudas G, Collins A T, Suhling K, Davies G and Heintzmann R 2009 J. Phys.: Condens. Matter 21364210

[21] Collins A T and Kiflawi I 2009 J. Phys.: Condens. Matter 21364209

[22] Steeds J W, Sullivan W, Wotherspoon A and Hayes J M 2009 J. Phys.: Condens. Matter 21364219

[23] Fisher D, Sibley S J and Kelly C J 2009 J. Phys.: Condens. Matter 21364213

[24] Bangert U, Barnes R, Gass M H, Bleloch A L, and Godfrey I S 2009 J. Phys.: Condens. Matter 21364208

[25] Khan R U A, Martineau P M, Cann B L, Newton M E and Twitchen D J 2009 J. Phys.: Condens. Matter 21364214

[26] Stoneham A M, Harker A H and Morley G W 2009 J. Phys.: Condens. Matter 21364222

[27] Evans D A, Roberts O R, Williams G T, Vearey-Roberts A R, Bain F, Evans S, Langstaff D and Twitchen D J 2009 J. Phys.: Condens. Matter 21364223

[28] Stoneham A M 2004 Nat. Mater. 33

[29] Berman R (ed) 1965 Physical Properties of Diamond (Oxford: Clarendon)

[30] Field J E (ed) 1979 The Properties of Diamond (London: Academic)

[31] Field J E (ed) 1992 The Properties of Natural and Synthetic Diamond (London: Academic)

[32] Sussmann R S (ed) 2009 CVD Diamond for Electronic Devices and Sensors (Wiley Series in Materials for Electronic and Optoelectronic Applications) (New York: Wiley) 